\section{Zeit der Verletzbarkeit}

Dass Kinder, die an Krebs erkranken, oft ein Leben lang unter den Folgen der Krankheit und der Therapie leiden, ist bekannt. Weniger weiß man über das Schicksal Jugendlicher und junger erwachsener Patienten mit Malignomen.

n der Childhood Cancer Survivor Study wurde anhand des umfassenden Registers der besondere Fokus auf die psychischen und neurokognitiven Funktionen jener langfristig überlebenden Patienten gelegt, die im Alter zwischen 11 und 21 Jahren an Krebs erkrankten. Für die aktuelle Analyse wurden Fragebögen von 6.192 Krebsüberlebenden und 390 Geschwistern ausgewertet. Unter den Überlebenden erhielten 1.589 ihre Diagnose im Alter zwischen 11 und 21 Jahren. Adjustiert für ihr Alter und Geschlecht, berichteten die früheren Krebspatienten häufiger von Depressionen als die Geschwistergruppe (11,7 vs. $8,0 \%$; Odds Ratio [OR] 1,55), häufiger von Ängstlichkeit (7,4 vs. 4,4\%; OR 2,00) und auch öfter von größeren Problemen, Aufgaben zu bewältigen (17,2 vs. 10,8\%; OR 1,72). Zudem erlebten sie ihre Emotionskontrolle (19,1 vs. $14,1 \%$; OR 1,74$)$ und ihre Gedächtnisleistung (25,9 vs. $19,0 \%$; OR 1,44) im Vergleich zur Geschwistergruppe als weniger zufriedenstellend. Zwischen Überlebenden, die eine Leukämie oder einen Tumor des Zentralnervensystems vor dem 11. Lebensjahr oder während der späten Adoleszenz hatten, gab es nur geringe Unterschiede im Ergebnis. Nur bei der Ängstlichkeit war die Gruppe der Älteren deutlich stärker betroffen (OR 1,44). Dagegen berichteten die Patienten dieser Altersgruppe mit einem Lymphom oder Sarkom insgesamt von weniger psychosozialen oder neurokognitiven Problemen als die bei der Diagnose jüngeren Patienten. Darüber hinaus waren Patienten, die über mangelhafte Fähigkeiten bei der Aufgabenbewältigung berichteten, häufiger arbeitslos (OR 2,93), hatten seltener einen College-Abschluss (OR $1,31)$ und lebten häufiger unselbstständig

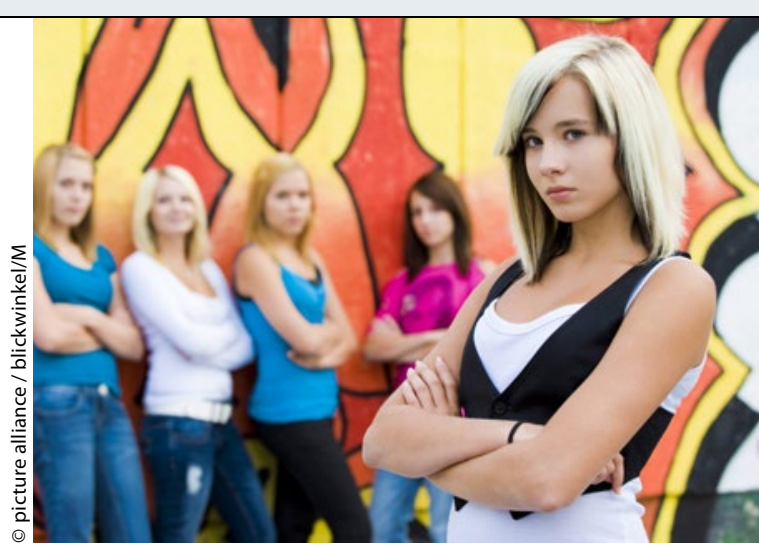

Eine Krebsdiagnose in der Adoleszenz kann schwerwiegende psychosoziale Folgen haben.

(OR 2,82). Mit einer höheren Arbeitslosigkeit gingen Somatisierung (OR 2,29) und Depressionen (OR 1,48) einher.

Fazit: Adoleszenz und frühes Erwachsenenalter sind kritische Phasen der Entwicklung. Eine onkologische Diagnose in dieser Zeit kann das Leben der Patienten psychisch und sozial nachhaltig beeinflussen.

Christina Berndt

Prasad PK et al. Psychosocial and Neurocognitive Outcomes in Adult Survivors of Adolescent and Early Young Adult Cancer: A Report From the Childhood Cancer Survivor Study. J Clin Oncol. 2015;33(23):2545-52.

\title{
Krebskranke Eltern sorgen sich um Erziehungskompetenz
}

\author{
Kinder, deren Eltern an Krebs \\ erkrankt sind, haben ein erhöhtes \\ Risiko für psychosoziale Probleme. \\ Eltern, die an Krebs erkanken, haben \\ Bedenken, dass sie ihre elterlichen \\ Aufgaben nicht ausreichend \\ wahrnehmen können.
}

\footnotetext{
E in Team um Cynthia W. Moore unterEuchte den Zusammenhang zwischen der Krebserkrankung eines Elternteils, der Lebensqualität und Erziehungsfunktionalität sowie den Ansichten der Eltern zu ihrer elterlichen Kompetenz und der Belastung der Kinder. 194 erwachsene, ambulante Krebspatienten mit Kindern bis zu einem Alter von 18 Jahren beantworteten Fragebögen zu ihrer gesundheitsbezogenen Lebensqualität (FACT-G, "functional assessment of cancer therapy: general"), zu Depressionen und Angst-
}

symptomen (HADS, „hospital anxiety and depression scale"), zu ihrer Gesamtbelastung (Distress Thermometer) und zu ihren Ansichten und Bedenken hinsichtlich ihrer elterlichen Kompetenz (,parenting concerns questionnaire").

$60 \%$ der befragten Patienten waren der Ansicht, dass sie die Bedürfnisse ihrer Kinder vor ihrer Krebsdiagnose extrem gut erfüllten, aber nur $11 \%$ gaben an, dies auch nach der Krebsdiagnose noch zu tun. Die mittleren Scores für den Glauben an ihre elterliche Kompetenz sanken nach der Diagnose signifikant. Diese Abnahme korrelierte mit einer zunehmenden Zahl an Klinikaufenhalten, der Behandlung mit einer systemischen Chemotherapie in den letzten Monaten, einer schlechteren gesundheitsbezogenen Lebensqualität und der Zunahme von Depression und Gesamtbelastung. Patienten mit den meisten Bedenken hin- sichtlich des Einflusses ihrer Krebserkrankung auf die Stimmung, körperliche Einschränkungen und Veränderungen im Tagesablauf ihrer Kinder hatten am stärksten den Glauben an ihre Kompetenz als Eltern und an die Kompetenz des anderen Elternteils verloren. Darüber hinaus hatten diejenigen Patienten mit der größten Abnahme ihres Glaubens an ihre elterliche Kompetenz die größten Bedenken, was die psychischen Belastungen ihrer Kinder durch die Krebserkrankung anging.

Fazit: Die Ergebnisse dieser Studie heben hervor, wie wichtig es ist, die Bedenken von krebskranken Eltern bezüglich ihrer Erziehungskompetenz anzusprechen.

Judith Neumaier

Moore CW et al. Parenting changes in adults with cancer. Cancer. 2015;121(19):3551-7. 